\title{
Preconditioning with Thyroid Hormones Improves Renal Functions Via Poly (ADP-Ribose) Polymerase-1 Reduction in a Rat Model of Renal Ischemia-Reperfusion Injury
}

\author{
HEND ASHOUR, M.D. ${ }^{1},{ }^{2}$; SOMIA A. SOLIMAN, M.D. ${ }^{3}$; HEBA MORSI, M.D. ${ }^{4}$ and \\ MARWA AL-KAFFAS, M.D. ${ }^{4}$ \\ The Departments of Physiology, Faculty of Medicine, Cairo University, Egypt ${ }^{1}$ and King Khalid University, Abha, KSA ${ }^{2}$, \\ The Departments of Pathology 3 and Medical Biochemistry \& Molecular Biology ${ }^{4}$, Faculty of Medicine, Cairo University, Egypt
}

\begin{abstract}
Background: Ischemia-Reperfusion Injury (IRI) is one of the major triggers of Acute Kidney Injury (AKI). AKI represents an important economic burden that is associated with high mortality and morbidity rates.
\end{abstract}

Aim of Study: This study focused on the role of poly (ADP-ribose) polymerase-1 (PARP-1) in acute renal ischemiareperfusion injury in case of preconditioning with thyroid hormone.

Material and Methods: Forty adult male wistar albino rats were divided equally into; Group I: Sham-operated for renal IRI, Group II: Euthyroid rats subjected to IRI, Group III: PARP-1 inhibitor (3-AB) was administered to the IRI rats aiming to confirm the PARP-1 role in mediating the renal ischemic injury (IRI-3-AB). Group IV preconditioned rats with a single eltroxin dose (100ug/ $/ \mathrm{kg} / \mathrm{ip})$ six hours before IRI (precond-IRI).

Results: PARP-1 inhibitor was associated with a significant improvement in the all measured renal function parameters, concomitant with reduced renal tissue inflammatory and oxidative stress markers and Apoptosis Inducing Factor (AIF) levels, indicating the intermediary role of PARP-1 in the renal ischemic injury.

The preconditioning with thyroid hormones resulted in a significant improvement in the renal functions (without elevation in the thyroid hormone level), via reduction in the inflammatory and oxidative stress markers induced by the IRI, this was confirmed by the histological assessment. The PARP-1, AIF and caspase- 3 overexpression were aborted and the ATP level was preserved.

The correlations between PARP-1 and renal function parameters and the apoptotic markers may explain its contribution in the pathogenesis of renal IRI condition.

Conclusion: These results suggest an important role of the preconditioning with thyroid hormone in amelioration of

Correspondence to: Dr. Hend Ashour, The Departments of Physiology, Faculty of Medicine, Cairo University, Cairo, Egypt and King Khalid University, Abha, KSA the oxidative stress, inflammation and PARP-1 overactivation in the renal injury induced by IRI.

Key Words: AKI - PARP-1 - Thyroid hormones.

\section{Introduction}

ACUTE Kidney Injury (AKI) is a serious lifethreatening clinical setting emerged as a major public health problem. AKI leads to decreased survival and increased progression of underlying Chronic Kidney Disease (CKD) if the patients survive [1]. Renal Ischemia-Reperfusion (IRI) injury is one of the major causes of Acute Kidney Injury (AKI) which can manifest histologically as Acute Tubular Necrosis (ATN). The cellular and molecular responses of the kidney to acute ischemic injury are complex and not fully understood [2]

High concentrations of Reactive Oxygen Species (ROS) are generated in the ischemic kidneys after reperfusion which can damage cellular components such as DNA, proteins, and lipids, compromising the integrity of the glomerular and tubular epithelium, which result in the development of ATN [3]. Accumulation of DNA breaks induces the poly (ADP-ribose) polymerase-1 (PARP-1) expression [4].

PARP-1 is a nuclear protein that normally regulates gene transactivation as a transcription coactivator and protein function via poly (ADPribosylation). PARP-1 activation is important for DNA repair. However, its excessive activation results in ATP depletion and metabolic crisis, inducing necrotic cell death [5]. In the same context, PARP-1 inhibition improved the outcome of multiple ischemic tissues including kidney [6-8]. The 
thyroid hormones are thought to be involved in the regulation of the PARP- 1 activities [4].

The relationship between kidney and thyroid function has been well known for many years. Thyroid Hormones (TH) directly affect the kidney influencing renal growth and development, Glomerular Filtration Rate (GFR), renal transport systems, sodium and water homeostasis.

Previous studies have demonstrated that administration of thyroid hormones before ischemic insults reduces the ischemia reperfusion injury $[9,10]$ however, the relation to PARP- 1 is still unclear.

The aim of this study is to elucidate the effect of preconditioning with thyroid hormones in the renal ischemia-reperfusion injury and its possible role in the regulation of the PARP-1.

\section{Material and Methods}

1- Experimental animals and study protocol: Experimental protocol and procedures used in this study were carried out in accordance with the international guidelines for the care and use of laboratory animals according to the National Institute of Health (NIH) protocol. The study was conducted in the duration between June and December 2018.

A total of 40 male Wistar albino rats $180-200 \mathrm{gm}$ provided by laboratory Animal House Unit of Faculty of Medicine, Cairo University were used. Throughout the study, the rats were kept under the same environmental conditions with free access to ordinary rat chow and water at normal day time and dark cycle. Prior to grouping, the euthyroid state was confirmed by analysis of thyroid profile (free T4 and TSH) for all animals. Animals were allowed to acclimatize their environment for seven days. Animals then were designated randomly into the following four groups (10 rats in each group), Group I: Sham-operated for renal IRI (Sham). Group II: Rats subjected to renal IschemiaReperfusion Injury (IRI): Normal saline ( $1 \mathrm{ml} / \mathrm{kg} / \mathrm{ip}$ ) was injected intraperitoneally (ip) as a vehicle. Group III: The PARP-1 inhibitor (3-amino benzamide)-obtained from (Sigma, Cairo, Egypt), was injected in a dose of $10 \mathrm{mg} / \mathrm{kg} / \mathrm{ip}$ [11] in twice doses; one dose was administered $6 \mathrm{~h}$ before the IRI induction and the second dose was introduced immediately after reperfusion. Group IV: (PrecondIRI), six hours before renal ischemia, the animals were preconditioned with a single Eltroxin dose (100ug/kg/ip) (Eltroxin 50mcg, GlaxoSmithKlinegsk Co. Cairo, Egypt) [9]
2- Induction of renal ischemia-reperfusion injury: The animals were injected with heparin 100U/Kg (Nile Co., Egypt) subcutaneously to prevent clotting before reperfusion then anesthetized by $50 \mathrm{mg} / \mathrm{kg}$ ketamine with $10 \mathrm{mg} / \mathrm{kg} / \mathrm{i} . \mathrm{p}$ xylazine hydrochloride (Sigma, Egypt) [2] . Under aseptic precautions, bilateral renal IRI was performed through a $2 \mathrm{~cm}$ midline laparotomy incision, the intestines were retracted to expose the renal pedicles. Microvascular clamps were placed around first the left and then the right renal pedicles blanching of the kidneys was observed. The total clamp time was 45 min during which the intestines were covered in warm and moist gauze. Afterward, the clamps were removed and the restoration of blood flow into the kidneys was confirmed visually. The intestines were returned into the abdominal cavity and the incision was then closed in layers [2]. Sham-operated animals underwent the same procedure with no occlusion of the renal pedicles. The animals were allowed to recover for two hours under observation in their cages. The animals then were placed in metabolic cages for $24 \mathrm{~h}$ urine collection with free access to food and water.

3-Measurement of arterial blood pressure and body weight assessment: A non-invasive arterial blood pressure systolic (SABP) and diastolic (DABP) was measured using a blood pressure transducer (ADInstruments), and the data was analyzed using LabChart 7 software. The body weight was determined using the digital weighing machine (Mettler-Toledo, 200).

4- Sample collection: Following the $24 \mathrm{~h}$ urine collection, the urine volume was measured and then centrifuged to separate debris. The urine samples were further analyzed for assessment of urinary creatinine and albumin.

Under 50mg/kg ketamine and $10 \mathrm{mg} / \mathrm{kg} / \mathrm{i} . \mathrm{p}$ xylazine anesthesia, blood samples were collected from the tail vein for assessment of Blood Urea Nitrogen (BUN), plasma creatinine and the thyroid profile levels for all animals.

5- Plasma and urine sample analysis: Plasma and urine levels of creatinine and BUN concentration were determined using commercially available kits (BioAssay Systems, Hayward, CA). Urine levels of albumin were determined using a commercially available rat albumin ELISA (Immunology Consultants Laboratory, Newberg, OR). Free serum T4 and TSH were estimated by Thyroxine (T4) (Mouse/Rat) ELISA Kit and the rat Thyroid Stimulating Hormone (TSH) ELISA Kit catalog number: CSB-E05115r according to provided protocol. 
6- Calculation of estimated GFR (eGFR): Estimated GFR was calculated using the following formula [12]:

eGFR $(\mathrm{ml} / \mathrm{min} / \mathrm{kg})=$ Urinary Creatinine $(\mathrm{mg} / \mathrm{dl})$ X Urine volume $(\mathrm{ml})$ X $1000(\mathrm{~g})$ Plasma creatinine (mg/dl) X Body weight (g) X 1440 (min).

Animals were killed immediately after samples collections by cervical dislocation under pentobarbital $(150 \mathrm{mg} / \mathrm{kg}$ i.p.) anesthesia. Tissue samples from the left kidneys were dissected for histopathological examination, and the right ones were used for measuring the following parameters: Malondialdehyde (MDA), Superoxide Dismutase (SOD), Neutrophil Gelatinase-Associated Lipocalin (NGAL), Activating Protein 1 (AP-1), Caspase 3, ATP, poly ADP-Ribose Polymerase (PARP-1) activity in the kidney.

7- Determination of Caspase 3, angiotensin II, NGAL and ATP: Caspase 3 and NGAL were determined by Rat ELISA Kit supplied by MyBiosource, USA according to manufacturer's instruction. ATP was determined by ATP Determination Kit (A22066) Molecular ProbesTM Luciferase Techonology. Angiotensin II was also measured by ELISA Kit, provided by CUSABIO, USA.

8- Determination of MDA and SOD: MDA levels were assessed for products of lipid peroxidation. MDA which is referred to as thiobarbituric acid-reactive substance, was measured with thiobarbituric acid at $532 \mathrm{~nm}$ in a spectrophotometer according to the method of [13]. The SOD activity was measured by determining the reduction of nitroblue tetrazolium by superoxide anion produced with xanthine and xanthine oxidase [14].

9- Immunoblotting detection of PARP-1: Immunoblotting was performed to detect PARP-1 protein expression. In brief, protein samples were electrophoretically separated on $10 \%$ polyacrylamide gels and transferred to nitrocellulose membranes, which were blocked overnight in 5\% nonfat dry milk diluted in Tris-buffered saline with $0.1 \%$ Tween20 , and then incubated for $1 \mathrm{~h}$ at room temperature with antibodies against PARP-1 (1:500) and bactin (Sigma Aldrich; 1:5000). After being washed, the membrane was incubated for $2 \mathrm{~h}$ with the secondary antibody (Santa Cruz Biotechnology; 1:2000) and subjected to a chemiluminescence detection system.

10- Quantitative real-time PCR for detection of $A P-1$ and AIF: Real-time PCR was performed for quantitative genes expression for AP- 1 and AIF. Tissue samples of all the studied groups were homogenized and total RNA was isolated with
RNeasy purification reagent (Qiagen, Valencia, CA). The purity of total RNA was measured with a spectrophotometer and the wavelength absorption ratio $(260 / 280 \mathrm{~nm})$ was between 1.8 and 2.0 for all preparations. Reverse transcription of total RNA to cDNA was carried out with a reverse transcription reaction (Superscript II, Gibco Life Technologies, Grand Island, NY, USA). Real-time PCR amplification and analysis were carried out using an Applied Biosystem with software version 3.1 (StepOneTM, USA). The reaction contained SYBR Green Master Mix (Applied Biosystems). The data were analyzed with the comparative Cycle Threshold (CT) method. The expression of 3 -actin mRNA was used as an internal control in all samples. The primers used were, for AP-1 forward; 5«-GTAATACGACTCACTATAGGGC-3 and reverse 5-ACTATAGGGCACGCGTGGT-3. The primer used for AIF detection is the forward primer 5-CCCCGATGTTGGCTATGA-3 and reverse primer 5-TCCTGACTGCTCTGTGGC-3. And for beta-actin we used forward primer: 5'GACGGCCAGGTCATCACTAT-3', reverse primer: 5'-CTTCTGCATCCTGTCAGCAA -3'.

11-Histopathological examination of the kidney tissue: The left kidneys were fixed in $4 \%$ paraformaldehyde and embedded in paraffin. Five tm sections were obtained and stained with hematoxylin and eosin (H \& E). The $\mathrm{H} \& \mathrm{E}$ sections were used in assessing the interstitial edema, inflammation, tubular and the epithelial damage at magnification X 400 , blindly by an expert pathologist. The degree of renal damage was scored using the scoring system for renal injury as reported by [15]. The scoring system consists of histological damage in 4 individual components endothelial, glomerular, tubular, and interstitial (EGTI scoring system).

\section{2- Statistical analysis:}

Comparisons between groups were done using the software statistical package SPSS (IBM. Chicago, IL, 2011). After performing the test of normality, one-way analysis of variance (ANOVA) followed by Tukey's post hoc test for multiple comparisons between the normally distributed variables, the data were described using mean and standard deviation. For comparing the histological scoring system, Kruscal-Wallis test and MannWhitney post hoc test were performed and represented as median and interquartile range. Pearson correlation coefficient was done to test for linear relations between PARP-1 and other variables. A probability value $p$-values less than 0.05 were considered as statistically significant. 


\section{Results}

\section{1- Thyroid profile analysis:}

Induction of IRI is considered as a stress condition, in which a small insignificant difference was detected regarding free T4 and TSH. Neither PARP-1 inhibitor nor preconditioning with a single thyroid dose revealed a significant change in the thyroid profile compared to the IRI group. Increased T4 level was detected between the treated groups (Table 1).

\section{2- Hemodynamic changes:}

The constricted renal vessels during ischemia in the IRI group resulted in a significant $(p<0.05)$ elevation in the angiotensin II levels which in turn raised the arterial blood pressure measurements. The group of PARP- 1 inhibitor and the thyroid preconditioned group expressed no significant change in the measured hemodynamic parameters compared to the IRI group (Table 1).

\section{3- Oxidative stress markers involvement in the different groups:}

The oxidative stress was increased in the kidney tissues of the IRI group, there was a significant elevation $(p<0.05)$ in the pro-oxidant malondaldehide (MDA) and decline in the antioxidant Superoxide Dismutase (SOD) levels $(p<0.001)(81.5 \pm 7.6$ $\& 0.58 \pm 0.19)$ compared to the sham group (15.5 \pm $1.8 \& 3.02 \pm 0.22)$ respectively.
PARP-1 inhibition group showed a significant $(p<0.001)$ reduction in the MDA $(30.8 \pm 13.4)$ and elevation of the SOD $(2.6 \pm 0.39)$ compared to the corresponding data in the ischemic untreated group. In the preconditioned group there was an improvement $(p<0.05)$ in the MDA and SOD levels $(36 \pm 5.6$ $\& 1.9 \pm 0.3$ respectively) compared to the IRI untreated group. We noticed a significant higher level of SOD in the PARP-1 inhibition group compared to the thyroid hormone preconditioned group Fig. $(1 \mathrm{~A}, \mathrm{~B})$.

\section{4- The role of inflammatory markers:}

In the renal tissues of the ischemic group, we observed a significant elevated $(p<0.05)$ inflammatory markers, Activating Protein 1 (AP-1) and the Neutrophil Gelatinase-associated Lipocalin (NGAL), $(8.7 \pm 1.1 \& 3.4 \pm 0.4)$ respectively compared to the corresponding values of the sham group ( $1 \pm 0.01 \& 0.5 \pm 0.17)$ respectively.

The group received the PARP-1 inhibitor (3AB) expressed a significant $(p<0.001)$ decline in both AP- 1 and NGAL levels $(3.1 \pm 0.59 \& 0.69 \pm$ $0.19)$ respectively. A decline in both inflammatory markers was noticed in the preconditioned group $(3.4 \pm 1.01 \& 0.79 \pm 0.11)$ respectively. There was no significant difference between the preconditioned group and the PARP-1 inhibition group. The NGAL levels in the PARP-1 inhibition were similar to that of the control group Fig. (1C,D).

Table (1): The thyroid profile, hemodynamic effect and the renal functions in the studied groups.

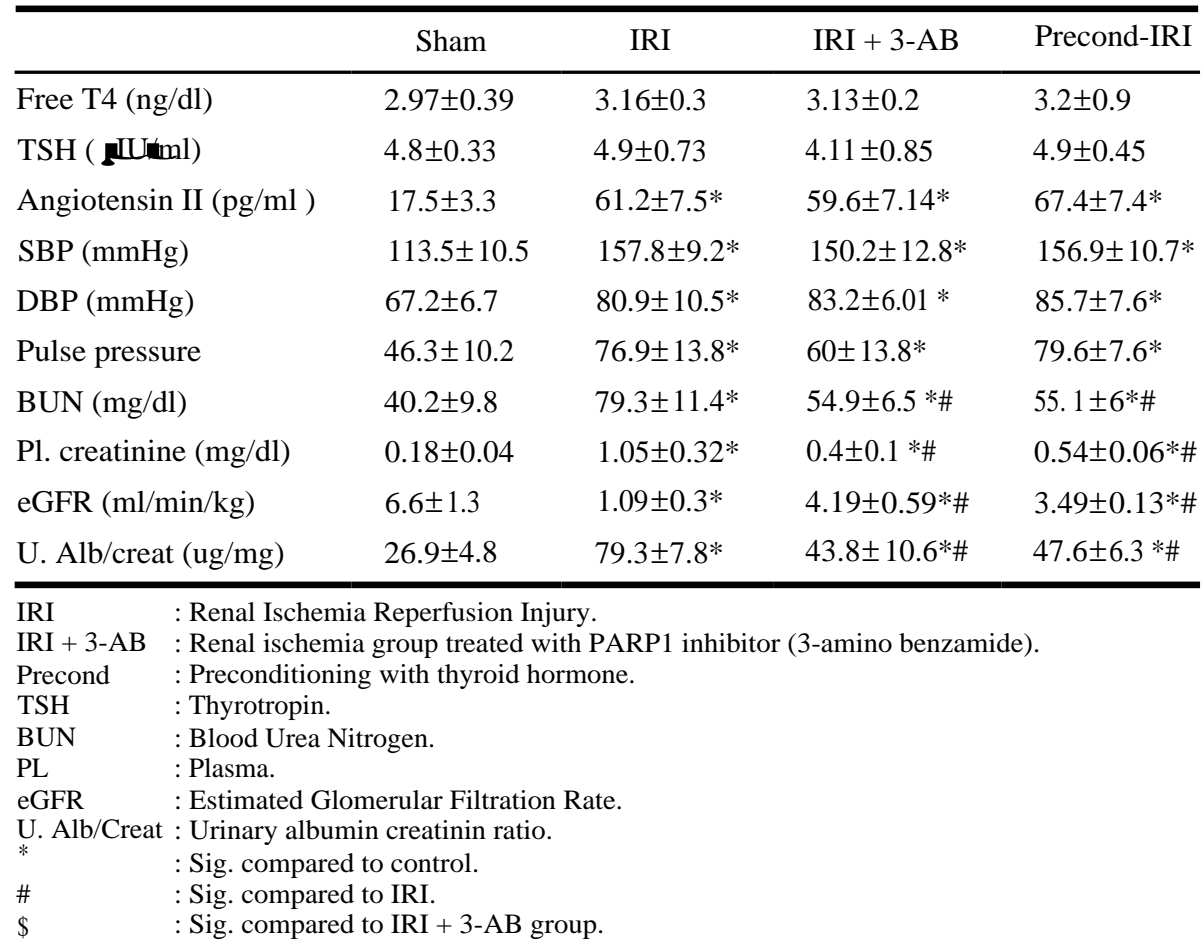



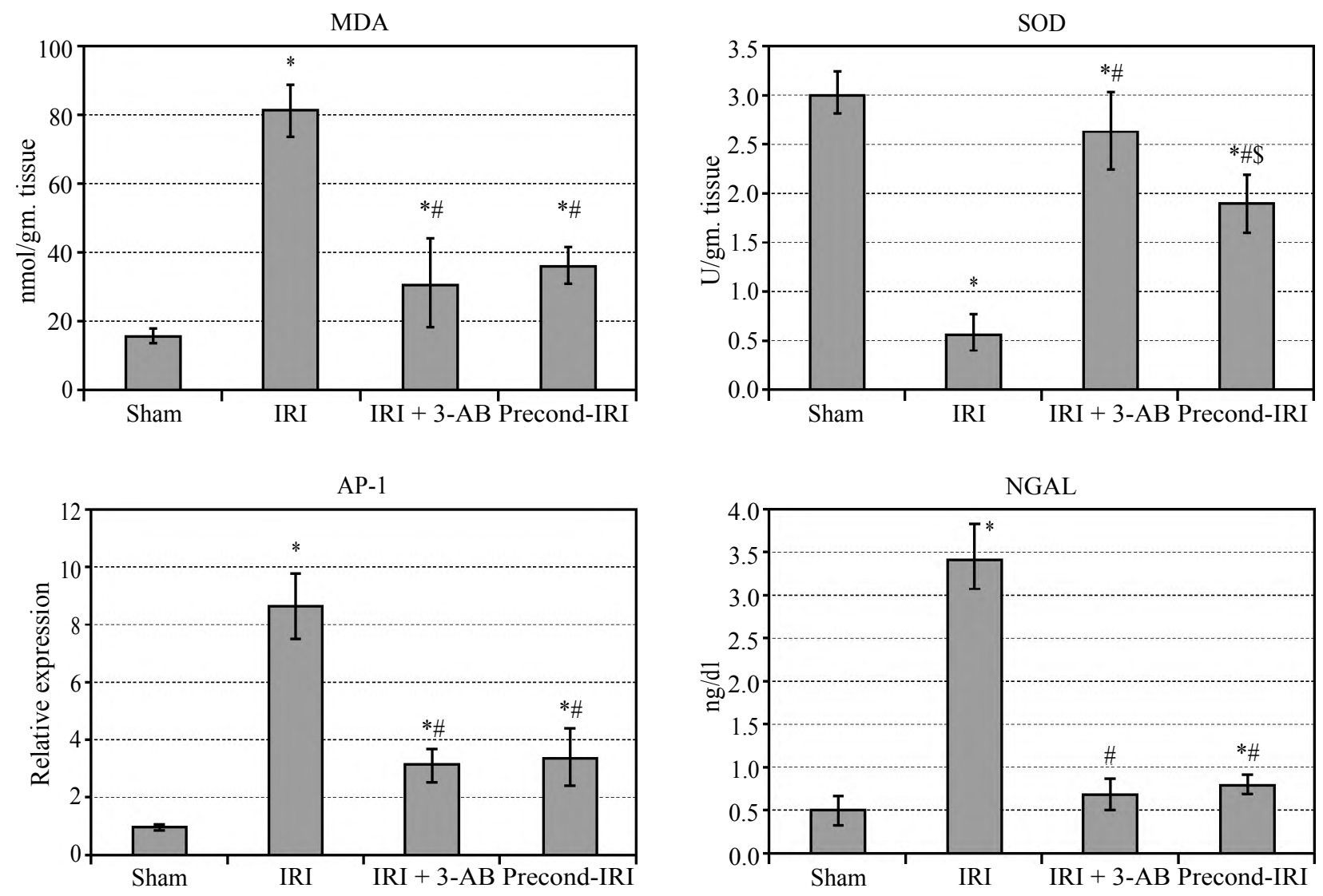

Fig. (1): The oxidative and inflammatory markers changes in different studied groups:

IRI : Ischemia-Reperfusion Injury.

IRI + 3-AB : Ischemic group treated with poly ADP-ribose polymerase (PARP-1) inhibitor (3-amino benzamide).

Precond-IRI : Preconditioned group with thyroid hormones.

A : Malondialdehyde (MDA).

B : Superoxide Dismutase (SOD).

C : Activating Protein 1 (AP-1).

D : Neutrophil Gelatinase-associated Lipocalin (NGAL).

* : Sig compared to control.

\# : Sig compared to IRI.

$\$$ : Sig compared to IRI + 3-AB group.

5- PARP-1, ATP and caspase-3 and ApoptosisInducing Factor (AIF) levels fluctuations in the studied groups:

Western blot of PARP- 1, and gene expression of AIF caspase- 3 were significantly induced ( $p$ $<0.001)$ in the kidney tissues of the IRI $(6.24 \pm 0.62$, $4.12 \pm 0.61 \& 11.22 \pm 1.9$ ) compared to the corresponding values in the sham-operated group (1.02 \pm $0.02,1.01 \pm 0.01 \& 2.4 \pm 0.78)$ respectively.

PARP-1 inhibition, resulted in decreased the elevated PARP-1 $(1.91 \pm 0.4)$ and the pro-apoptotic markers AIF and caspase-3 $(1.6 \pm 0.39 \& 8.4 \pm 1.49)$ respectively.

Pre-treatment with a single thyroid dose caused a significant $(p<0.001)$ decline in the readings of PARP-1, AIF and caspase-3 $(2.25 \pm 0.38,1.6 \pm 0.39$ $\& 3.4 \pm 0.37)$ respectively. This was associated with more caspase- 3 reduction compared to the IRI + 3-AB group that could be explained by the antiap- optotic effect of the thyroid hormone on the pathway rather than PARP-1 inhibition Fig. (2A-C).

During ischemic injury, the renal tissues consumption of ATP was increased leaving low ATP levels compared to the sham group. Either PARP1 inhibition or preconditioning with single thyroid hormone dose significantly $(p<0.05)$ preserved the ATP content in the kidney tissue compared to the IRI group Fig. (2D).

6- Renal function assessment in the studied groups:

The deterioration in the measured renal function parameters indicated the functional disturbance following ischemia-reperfusion injury in the IRI group.

PARP-1 pathway inhibition and preconditioning with thyroid hormone were resulted in renal functional improvement in all measured parameters, indicating a valuable role of renal tissue protection from ischemic injury by blocking the PARP-1 signaling pathway (Table 1). 
PARP-1

Beta actin
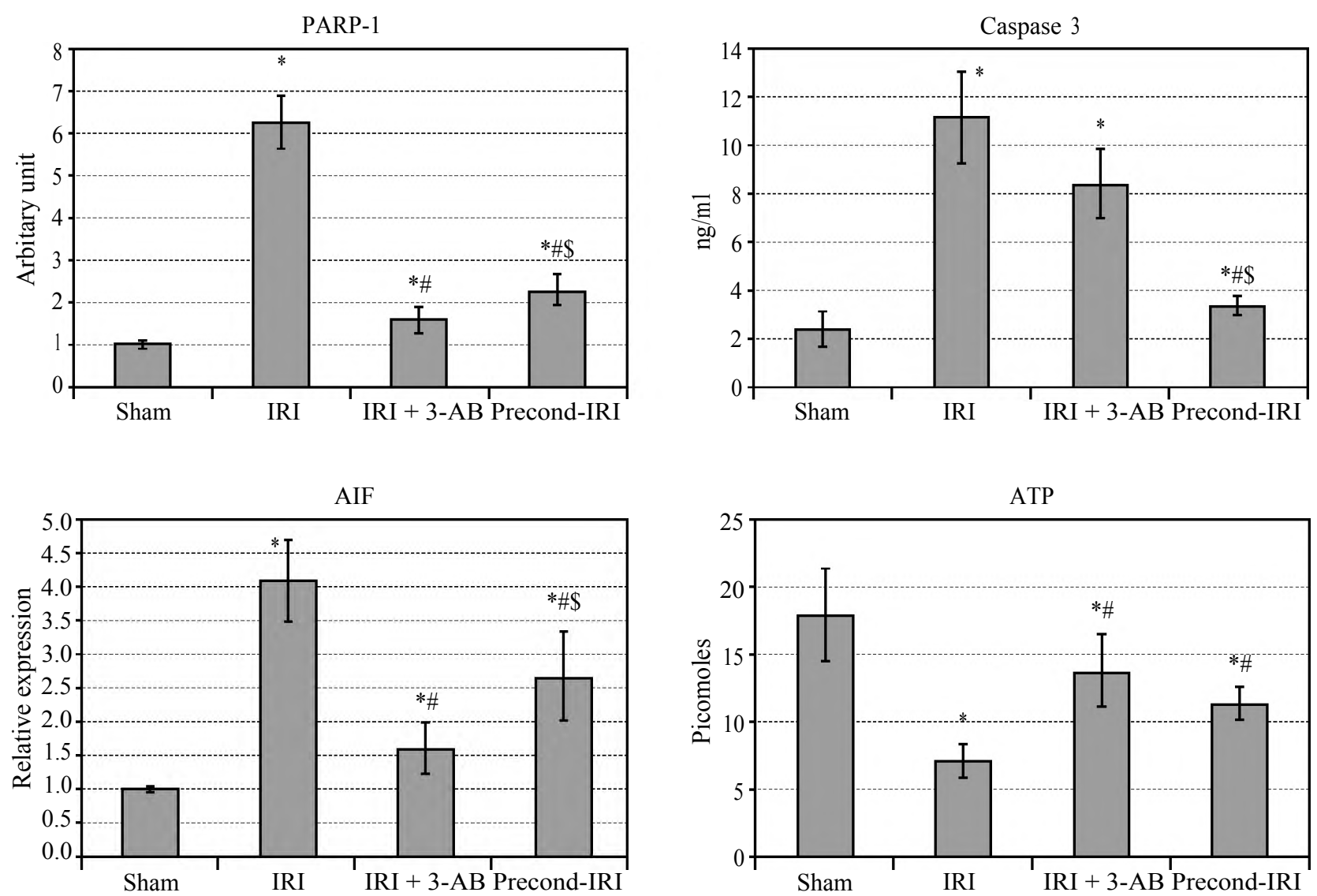

Fig. (2): Representation of A: Poly ADP-ribose polymerase (PARP-1), B: Apoptosis Inducing Factor (AIF), C: Caspase 3 and D: ATP levels in the kidney tissues in different groups.

IRI : Ischemia-Reperfusion Injury.

IRI + 3-AB : Ischemic group treated with PARP-1 inhibitor (3-amino benzamide) and the preconditioned group with thyroid hormones (Precond-IRI)

: Sig compared to control

: Sig compared to IRI.

: Sig compared to IRI + 3-AB group.

\section{7- Histological finding evaluation:}

As shown in (Table 2) and Figs. $(3,4)$, the IRI group illustrated a significant increase $(p<0.01)$ in the total EGTI histology score (endothelial, glomerular, tubular and interstitial cell damage) when compared to sham group.

PARP-1 inhibition by 3-AB caused a significant reduced EGTI histological injury score as seen in Figs. $(4,5)$. The EGTI histology score was significantly improved $(p<0.05)$ in the thyroid hormone preconditioned group as compared to the ischemic non treated group.
Table (2): Histopathological assessment of the kidney tissues.

\begin{tabular}{lclll}
\hline & Sham & \multicolumn{1}{c}{ IRI } & IRI + 3-AB & Precond-IRI \\
\hline Endothelial & 0 & $1.5(1-2.25)$ & $2(1-2)$ & $1(0.75-1.25)$ \\
Glomerular & 0 & $2(2-2)$ & $1(0-1)$ & $1(0.75-2)$ \\
Tubular & 0 & $2.5(2-3)$ & $1(0-1)$ & $2(2-2.25)$ \\
Interstitial & 0 & $2(1.75-2.25)$ & $1(0-1)$ & $1(0.75-1.25)$ \\
\hline Total score & 0 & $8(7.75-9)^{*}$ & $5(4-5)^{* \#}$ & $5.5(4.75-6)^{* \#}$ \\
\hline
\end{tabular}

IRI : Renal Ischemia Reperfusion Injury.

IRI + 3-AB : Renal ischemia group treated with PARP1 inhibitor (3-Amino Benzamide).

Precond : Preconditioning with thyroid hormone.

: Sig compared to control.

\# $\quad$ : Sig compared to IRI. 

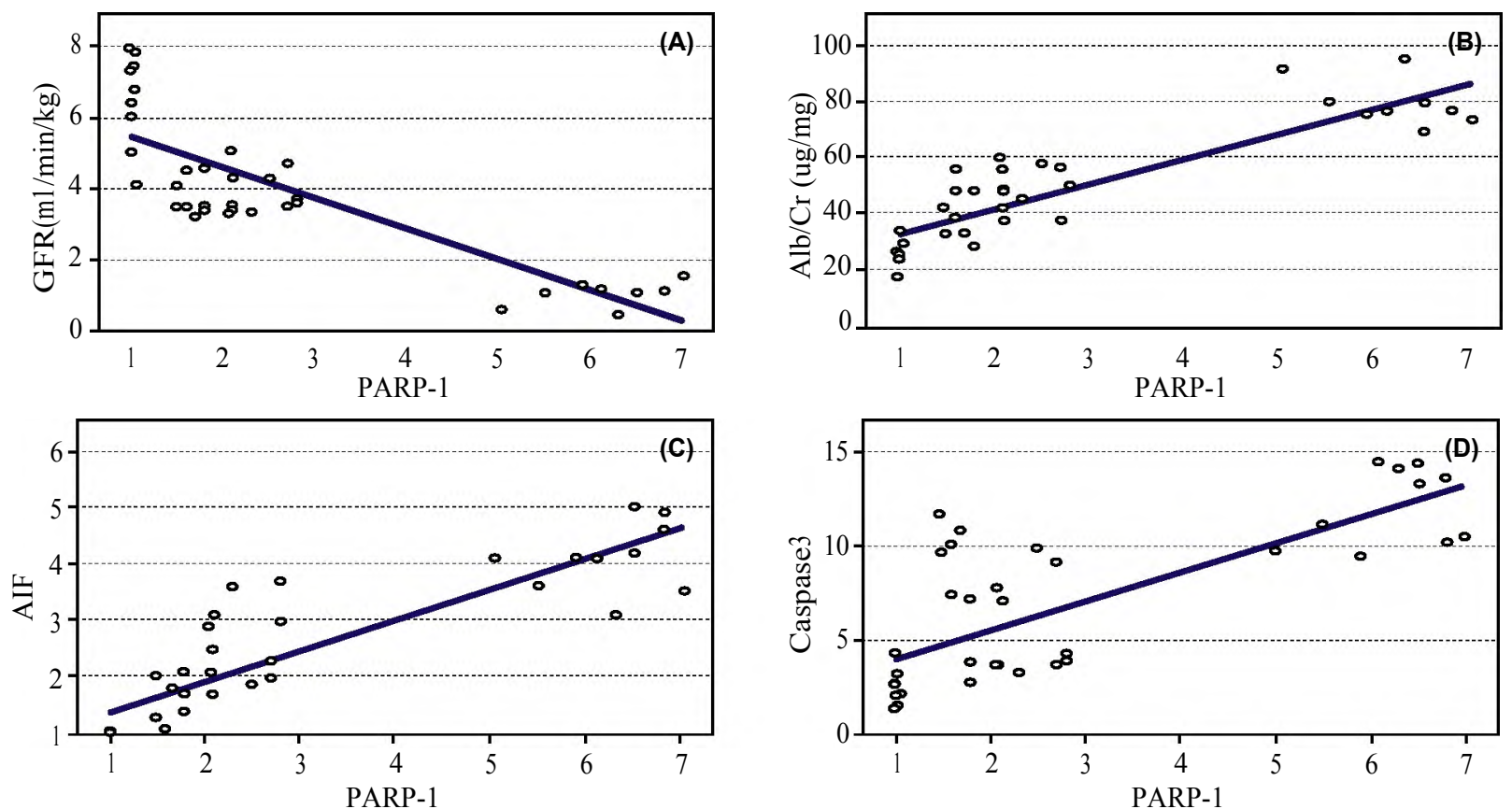

Fig. (3): PARP-1 correlation with the renal functions (eGFR and Alb/Cr ratio) as shown in Fig. (3A \& B) and the apoptotic factors (caspase-3 and AIF) represented in Fig. (3C \& D). The results gave a strong positive correlation with $\mathrm{Alb} / \mathrm{Cr}$ (ug/mg), caspase 3 and AIF ( $r=0.899,0.763$ and 0.889 respectively). A negative correlation gathered PARP-1 and eGFR $(r=0.839, p<0.001)$.
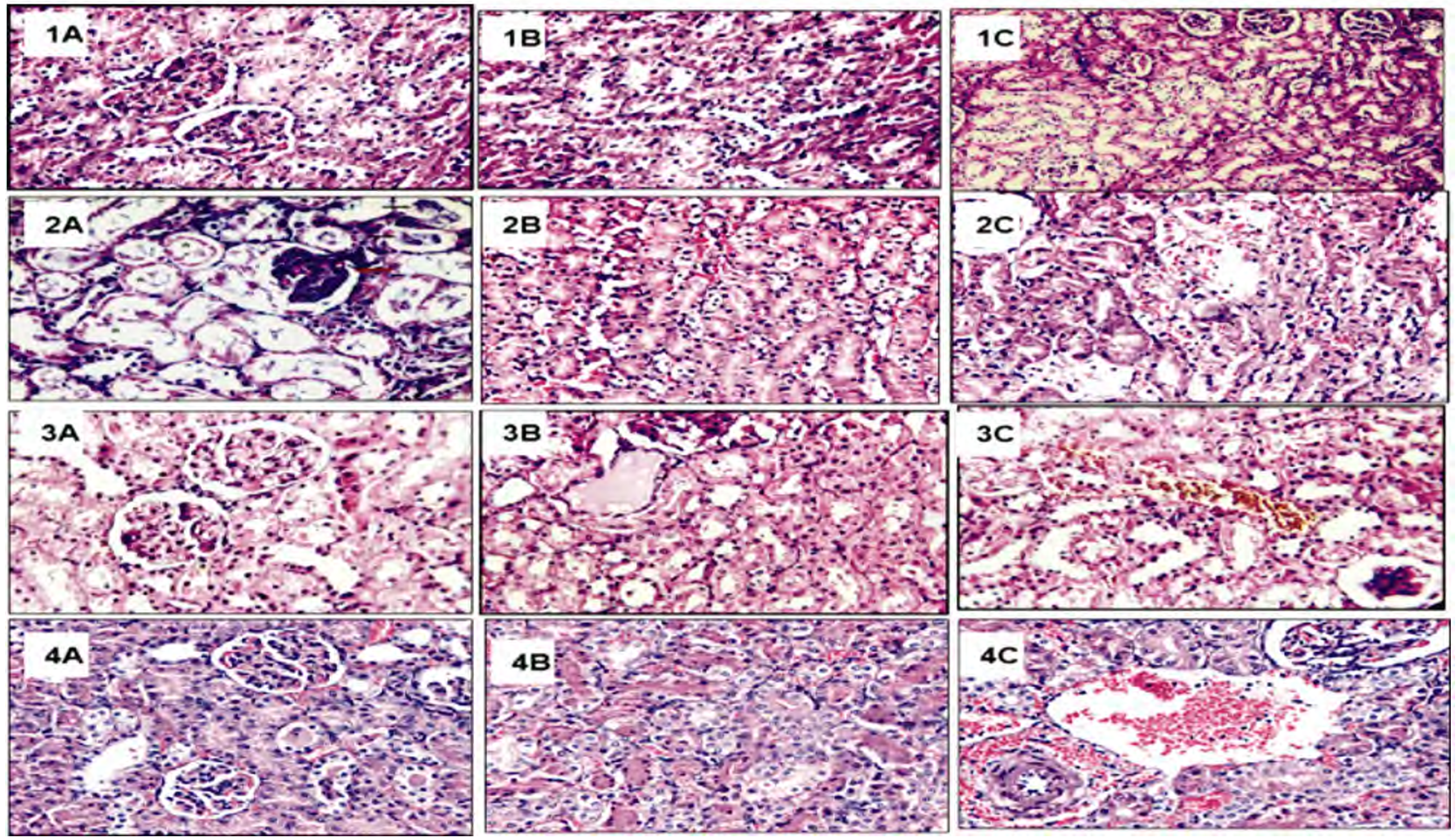

Fig. (4): Histological (H \& E) assessment of the kidney tissues (X400 original magnification) using the EGTI (endothelial, glomerular, tubular and interstitial) in the 1: Sham-operated group, 2: Renal ischemia-reperfusion injury, 3: IRI + 3-AB: Ischemic group treated with poly ADP-ribose polymerase (PARP-1) inhibitor (3-amino benzamide) and 4: Preconditioning with thyroid hormones. 1A, B \&C showing normal histological structure (score 0 ). In $2 \mathrm{~A}$ the stars show necrosis of renal tubules less than $30 \% \&$ the black arrow points tubular casts (score 3), the red arrow indicates glomerular tuft ischemic retraction with disruption of Bowman's capsule (score 2), 2B shows interstitial edema \& hemorrhage (score 1) and 2C endothelial disruption \& interstitial hemorrhage (score 2). The 3A slide demonstrates a normal glomerular structure with 0 score. In the $3 \mathrm{~B}$, the tubules loss of the brush border with hyaline cast formation giving a score 3 . Endothelial disruption with mild interstitial hemorrhage was seen in the $3 \mathrm{C}$ image. In $4 \mathrm{~A}$ image there is normal glomerular pattern (score 0), in 4B there is loss of brush borders together with tubular hyaline casts (score 2), 4C interstitial hemorrhage and edema (score 1) and the endothelial cells are disrupted (score 2). 


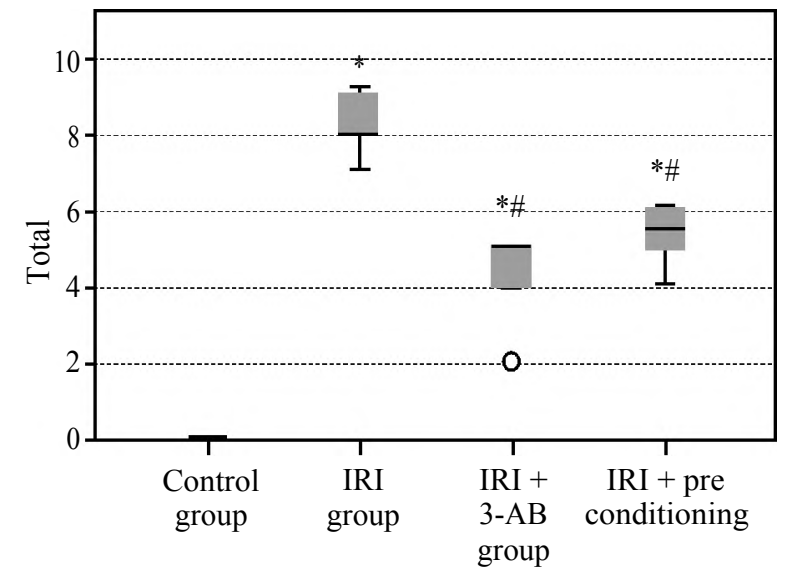

Fig. (5): Box blot represented the total EGTI renal injury score. Effect of ischemia-reperfusion injury (IRI), ischemic group treated with 3-amino benzamide (IRI + 3-AB) and a preconditioning with thyroid hormone on the total histology score EGTI (endothelial, glomerular, tubular and interstitial). The analysis presented as boxplots showing deteriorated renal score parameters in the ischemic, that was improved in both the IRI $+3-\mathrm{AB}$ and the preconditioned groups.

*: Sig compared to control.

\#: Sig compared to IRI.

$\$$ : Sig compared to IRI $+3-\mathrm{AB}$

\section{8- Correlation:}

Table (3) and Fig. (3) demonstrate the PARP1 correlation with the renal functions (eGFR and $\mathrm{Alb} / \mathrm{Cr}$ ratio) and the apoptotic factors (caspase3 and AIF). The results gave a strong positive correlation with caspase-3, Alb/Cr (ug/mg), caspase 3 and AIF. A negative correlation gathered PARP1 and eGFR indicating the involvement of PARP1 in the pathogenesis of AKI of the ischemic injury.

Table (3): Correlation between the level of PARP-1 and the levels of different renal functions parameters and the pro-apoptotic factors.

\begin{tabular}{ll}
\hline & PARP-1 \\
\hline$e G F R(\mathrm{ml} / \mathrm{min} / \mathrm{kg}):$ & -0.839 \\
$r$ & $<0.001$ \\
$p$-value & 40 \\
$\mathrm{~N}$ & \\
$A l b / C r(u g / m g):$ & 0.899 \\
$r$ & $<0.001$ \\
$p$-value & 40 \\
$\mathrm{~N}$ & \\
Caspase-3: & 0.763 \\
$r$ & $<0.001$ \\
$p$-value & 40 \\
$\mathrm{~N}$ & \\
$A I F$ : & 0.889 \\
$r$ & $<0.001$ \\
$p$-value & 40 \\
$\mathrm{~N}$ &
\end{tabular}

eGFR: Estimated GFR.

Alb/Cr: Albumin creatinine ratio.

AIF : Apoptosis Inducing Factor between the total number (40) of the studied group.

\section{Discussion}

The results suggest an important role of the thyroid hormones preconditioning in amelioration of oxidative stress, inflammation and PARP-1 overactivation induced by renal IRI. A positive correlation was found between PARP- 1 and the pro-apoptotic factors AIF and caspase-3 as well as urinary albumin creatinine ratio suggesting its deleterious effect on the renal Ischemia-Reperfusion Injury (IRI).

Several studies reported increased oxidative stress as a consequence of the renal IRI with elevated MDA and reduced SOD levels [16]. Generation of Reactive Oxygen Species (ROS) can trigger inflammation, and induce tubular cell apoptosis and necrosis [17]. Gelatinase-associated lipocalin (NGAL), is a new biomarker of kidney disease, that was elevated in acute ischemic and nephrotoxic injury a short time even before creatinine [15] Activating Protein 1 (AP-1) was also expressed and regulates cytokine gene expression in response to the ischemic stress [9].

ROS induce lipid peroxidation of membranes, protein denaturation, and DNA strand breaks. The massive DNA damage leads to excessive activation of the DNA repair enzyme PARP-1 and subsequent ATP depletion and metabolic disturbance $[12,13]$. Moreover, PARP-1 could induce a distinct form of cell death termed "parthanatos" [PAR + "thanatos," meaning death] independent of the caspase pathway. PARP-1 initiates cell death by mitochondrial Apoptosis-Inducing Factor (AIF) translocation to the nucleus [20]. AIF is a flavoprotein with a prosurvival role when it is situated in the mitochondria intermembrane space $[\mathbf{2 1 , 2 2}]$, whereas its release into the cytosol followed by nuclear translocation is considered as a powerful trigger of apoptosis. In case of parthanatos, AIF release occurs even earlier than that of cyt $\mathrm{c}$ and caspase activation [20]. When AIF is activated and translocated to the nucleus, it achieves a large-scale chromatin condensation and DNA fragmentation [23] and promotes the PARP-1-dependent cell death [24].

It is unclear how exactly could PAR polymers induce the release apoptosis-inducing factor from the mitochondria into the cytosol. However, energetic failure and PARP-1 hyperactivation and the PAR (poly (ADP-ribose) accumulation, resulted in translocation of PAR or perhaps a product of PAR catabolism, to the cytosol where it interacts with the outer mitochondrial surface increasing its permeability causing AIF from the mitochondria 
and subsequently parthanatos [25]. Recently, it was shown that PAR released in cytosol causes mitochondria $\mathrm{Ca} 2$ + disturbance, together with the increase in ROS production in the Electron Transport Chain (ETC) and dissipation of the mitochondrial membrane potential resulted in AIF release from the mitochondria [22]. In the same context, it was shown that the translocation of AIF into the nucleus was diminished in PARP- 1 deficient fibroblasts [24]. The PARP-1 response may be influenced by the cell type, as well as the stimuli type, strength, and duration $[\mathbf{2 6 , 2 7 ]}$.

The metabolic disturbance developed during renal IRI resulted in renal dysfunctions $[4,11]$. The histological findings supported the biochemical results, morphological changes in renal parenchyma, tubular necrosis with a severe detachment of epithelial cells from the basement membrane, loss of brush border, and intratubular casts was recorded in the IRI group $[4,7]$

The PARP- 1 inhibitor 3-AB administration in Group III was to confirm that the ischemic injury was mediated through the PARP-1 overexpression. And so, all measured inflammatory, apoptotic, oxidative stress markers and the apoptotic markers was resolved in the group received PARP-1 inhibitor compared with the ischemic group. This was verified by the improved renal function tests and the histological image analysis.

The administration of a single dose of thyroid hormone to rats at $6 \mathrm{~h}$ before IRI significantly reduced oxidative stress variable MDA and the expression of PARP- 1 and increased the level of SOD as compared to group IRI. These results were in agreement with [4,7] studies who examined the same dose at 6 h or 24 hours pre ischemia.

In the same context, it was shown that $\mathrm{T} 3$ preconditioning 36 hours preceding liver ischemia protected the liver against IRI-induced oxidative stress by reestablishing redox homeostasis [28] Three doses of thyroid hormone were associated with elevated superoxide dismutase level that inhibited kappaB-alpha phosphorylation and nuclear factor-kappaB activation [29].

However, unsuccessful administration of 3, 5diiodothyropropionic acid, 30min before renal IRI injury; may be due to short time course to exert preconditioning effect on oxidative stress [30]

PARP- 1 activity and caspase-3 level were decreased in case of preconditioning with thyroid hormone as compared to IRI group either in acute renal IRI [4] or in the chronic kidney disease [31]
PARP-1 ablation preserved ATP levels, renal functions, and attenuated inflammatory response preventing the progression of acute renal failure complications [8]

Pre-treatment with single T3 dose reduced the postischemic hepatic injury [32] and renal IRI [9] via inhibiting neutrophil infiltration and downregulation of the proinflammatory cytokines TNF- a, IL-1b, IL-6, and the transcription factors NF-1B and AP-1, and the adhesion molecules (ICAM-1 and VCAM-1). A cluster of proinflammatory cytokines and chemokines including serum levels of IL-6 and TNF-a were significantly lowered following $\mathrm{T} 3$ administration in the kidney remnants associated with amelioration in the renal functions [31], associated with diminution in the glomerular and tubular injury [9] .

In conclusion, preconditioning with $\mathrm{T} 3$ reduced the clinical and histological findings associated with preservation of ATP levels, explained by the changes in PARP-1 level. The correlations between PARP-1 and renal function parameters may clarify its contribution in the pathogenesis of the ischemic renal injury condition.

Conflict of interest: None is present.

\section{References}

1- SINGBARTL K. and KELLUM J.A.: AKI in the ICU: Definition, epidemiology, risk stratification, and outcomes. Kidney Int., 81: 819-25. Doi:10.1038/ki.2011.339, 2012.

2- OZBILGIN S., OZKARDESLER S., AKAN M., BOZTAS N., OZBILGIN M., ERGUR B.U., et al.: Renal Ischemia/ Reperfusion Injury in Diabetic Rats: The Role of Local Ischemic Preconditioning. Biomed Res. Int., Doi: 10.1155/ 2016/8580475, 2016.

3- FONSECA I., REGUENGO H., ALMEIDA M., DIAS L., MARTINS L.S., PEDROSO S., et al.: Oxidative Stress in Kidney Transplantation. Transplantation, 97: 1058-65. Doi: 10.1097/01. TP.000043 8626.91095.50, 2014.

4- FERREYRA C., VARGAS F., RODRÍGUEZ-GÓMEZ I., PÉREZ-ABUD R., O'VALLE F. and OSUNA A.: Preconditioning with Triiodothyronine Improves the Clinical Signs and Acute Tubular Necrosis Induced by Ischemia/ Reperfusion in Rats. PLoS One, 8: 2-10. Doi: 10.1371/ j ournal.pone.0074960, 2013.

5- SONG H., YOON S.P. and KIM J.: Poly (ADP-ribose) polymerase regulates glycolytic activity in kidney proximal tubule epithelial cells. Anat. Cell. Biol., 49: 79-87, 2016.

6- CRAWFORD R.S., ALBADAWI H., ATKINS M.D., JONES J.E., YOO H., CONRAD M.F., et al.: Post Ischemic PARP Inhibition Reduces Ischemia Reperfusion Injury in a Hind-Limb Ischemia Model. Surgery, 148: 110-8, 2011. Doi: 10.1016/j.surg..12.006.Post. 2009.

7- MORAL R.M.G., GÓMEZ-MORALES M., HERNÁNDEZ-CORTÉS P., AGUILAR D., CABALLERO T., 
ANEIROS-FERNÁNDEZ J., et al.: Lesion in Ischemia/ Reperfusion Renal Mouse Model after Cold Prolonged Ischemia. Sci. Journa; Article ID. Doi: http://dx.doi.org/ 10.1155/2013/486574, 2013.

8- ZHENG J., DEVALARAJA-NARASHIMHA K., SINGARAVELU K. and PADANILAM B.: Poly (ADP-ribose) polymerase- 1 gene ablation protects mice from ischemic renal injury. Am. J. Physiol. Ren. Physiol., 288: F387398, 2005, doi: 10.1152/ajprenal.00436.2003.

9- KIM S.M., KIM S.W., JUNG Y.J., MIN S.IL, MIN S.K., KIM S.J., et al.: Preconditioning with thyroid hormone (3, 5, 3-triiodothyronine) prevents renal ischemiareperfusion injury in mice. Surg. (United States), 155: 554-61. Doi: 10.1016/j.surg.2013.10.005, 2014.

10-ZAMAN J., JEDDI S., DANESHPOUR M.S., ZARKESH M., DANESHIAN Z. and GHASEMI A.: Ischemic postconditioning provides cardioprotective and antiapoptotic effects against ischemia-reperfusion injury through iNOS inhibition in hyperthyroid rats. Gene, 570: 185-90. Doi: 10.1016/j.gene.2015.06.011, 2015.

11- MARTIN D.R., LEWINGTON A.J.P., HAMMERMAN M.R., PADANILAM B.J., DIVISION R., DANIEL R., et al.: Inhibition of poly (ADP-ribose) polymerase attenuates ischemic renal injury in rats, 297: 1834-40, 2000.

12- RASHED L.A., ELATTAR S., ELTABLAWY N., ASHOUR H., MAHMOUD L.M. and EL-ESAWY Y.: Mesenchymal stem cells pretreated with melatonin ameliorate kidney functions in a rat model of diabetic nephropathy, 571: 564-71, 2018.

13- BUEGE J.A. and AUST S.D.: Microsomal Lipid Peroxidation. Methods Enzymol., 129: 302-10, 1975.

14- SUN Y., OBERLEY L. and YING L.: A Simple Method for ClinicalAssay of Superoxide Dismutase. Clin. Chem., 34: 497-500, 1988.

15- KHALID U., PINO-CHAVEZ G., NESARGIKAR P., JENKINS R.H., BOWEN T., FRASER D.J., et al.: Kidney ischaemia reperfusion injury in the rat: The EGTI scoring system as a valid and reliable tool for histological assessment. J. Histol. Histopathol., 3: 1. Doi: 10.7243/2055091X-3-1, 2016.

16- EREM C., SULEYMAN A.K., VAN N.C., MENTESE A., NUHOGLU I. and UZUN A.: Ischemia-modified albümin and malondialdehyde levels in patients with overt and subclinical hyperthyroidism: Effects of treatment on oxidative stress, 62: 493-501. Doi:10.1507/endocrj.EJ140542, 2015.

17- QIAO X., LI R., LI H., ZHU G., HUANG X., SHAO S., et al.: Intermedin protects against renal ischemiareperfusion injury by inhibition of oxidative stress: 112 9, 2012, doi: 10.1152/ajprenal.00054.2012.

18- CHIARUGI A.: Poly (ADP-ribose) polymerase: Killer or conspirator? The "suicide hypothesis" revisited. Trends Pharmacol Sci., 23: 122-9. Doi: 10.1016/S0165-6147 (00)01902-7, 2002

19- WANG L., LIU X., CHEN H., CHEN Z., WENG X., QIU T., et al.: Effect of picroside II on apoptosis induced by renal ischemia/reperfusion injury in rats. Exp. Ther. Med., 9: 817-22. Doi: 10.3892/etm.2015.2192, 2015.

20- ANDRABI S.A., DAWSON T.M. and DAWSON V.L.: Mitochondrial and Nuclear Cross Talk in Cell Death: Parthanatos. Ann. N. Y. Acad. Sci., 1147: 233-41. Doi: 10.1196/annals. 1427.0 14.Mitochondrial, 2008.

21- KIDNEYS F., DYSFUNCTION T. and SYNERGY A.U.: Failing Kidneys and Thyroid Dysfunction d An, 93: 5557. Doi: 10.1016/j.mayocp.2018.03.027, 2018.

22- MADUNGWE N.B., FENG Y., LIE M., TOMBO N., LIU L., KAYA F., et al.: Mitochondrial inner membrane protein ( mitofilin ) knockdown induces cell death by apoptosis via an AIF-PARP-dependent mechanism and cell cycle arrest, 28-43, 2018, doi: 10.1152/ajpcell.00230.2017.

23- WANG Y., DAWSON V.L. and DAWSON T.M.: Poly (ADP-ribose) Signals to Mitochondrial AIF: A Key Event in Parthanatos. Exp. Neurol., 218: 193-202. Doi: 10.1016/ j.expneurol.2009.03.020.Poly(ADP-ribose), 2010.

24- KIM M.Y., ZHANG T. and KRAUS W.L.: Poly (ADPribosyl) ation by PARP-1:PAR-laying'NAD into a nuclear signal. Genes Dev., 19: 1951-67. Doi: 10.1101/gad. 1331805. ity, 2005.

25- LINKERMANN A.: Nonapoptotic cell death in acute kidney injury and transplantation. Kidney Int., 89: 4657, 2016. Doi:10.1016/j.kint..10.008. 2015.

26- VIRAG I.: PARP-1 and the Shape of Cell Death In Poly (AD: Landes Biosci, 141-54, 2005.

27- WEAVER A.N. and YANG E.S.: Beyond DNA repair: Additional functions of PARP-1 in cancer, 3: 1-11. Doi: 10.33 89/fonc.2013.00290, 2013.

28- FERNANDEZ V., CASTILLO I., TAPIA G., ROMANQUE P., URIBE-ECHEVARRÍA S., URIBE M., et al.: Thyroid Hormone Preconditioning: Protection Against IschemiaReperfusion Liver Injury in the Rat. Hepatology, 45: 1707. Doi: 10.1002/hep.21476, 2007.

29- FERNÁNDEZ V., TAPIA G., VARELA P., CASTILLO I., MORA C., MOYA F., et al.: Redox up-regulated expression of rat liver manganese superoxide dismutase and $\mathrm{Bcl}-2$ by thyroid hormone is associated with inhibitor of $\mathrm{\kappa B}-\alpha$ phosphorylation and nuclear factor- $\mathrm{KB}$ activation. J. Endocrinol., 186: 539-47. Doi:10.1677/joe.1.06261, 2005.

30- HADI N.R., AL-AMRAN F.G. and HUSSEIN A.A.: Effects of thyroid hormone analogue and a leukotrienes pathway-blocker on renal ischemia/reperfusion injury in mice, 1-11, 2011

31- AGATY S.M. El.: Triiodothyronine attenuates the progression of renal injury in a rat model of chronic kidney disease. Can J. Physiol. Pharmacol., 29: 1-26. Doi:10.1164/ rccm.200909-14200C, 2018.

32- TAKI-ELDIN A., ZHOU L., XIE H.Y., CHEN K.J., YU D., HE Y., et al.: Triiodothyronine attenuates hepatic ischemia/reperfusion injury in a partial hepatectomy model through inhibition of proinflammatory cytokines, transcription factors, and adhesion molecules. J. Surg. Res., 178: 646-56. Doi: 10.1016/j.jss.2012.05.069, 2012. 


\section{تعمل التهيئة الأولية بهرمونات الغدة الدرقية على تحسين وظائف الكُلى

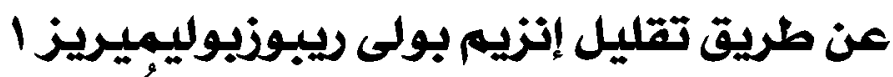

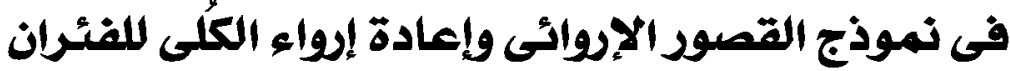

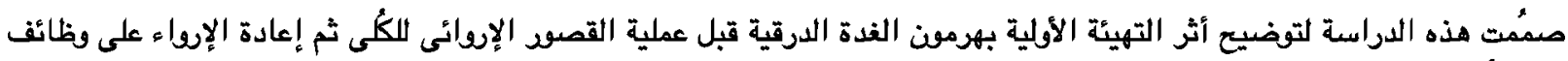

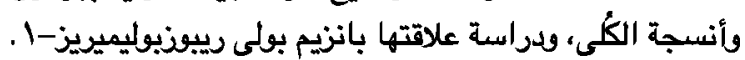

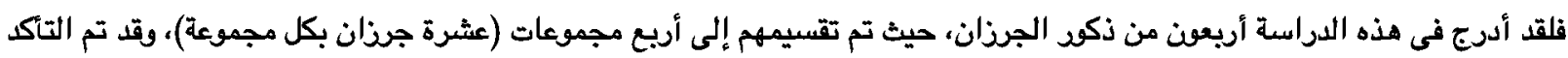

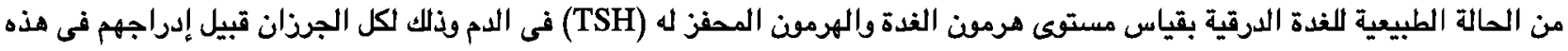

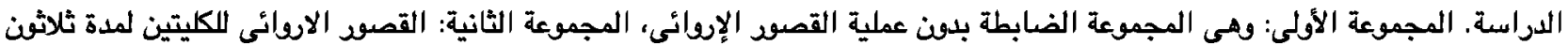

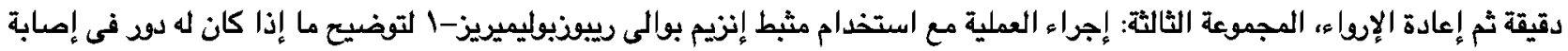

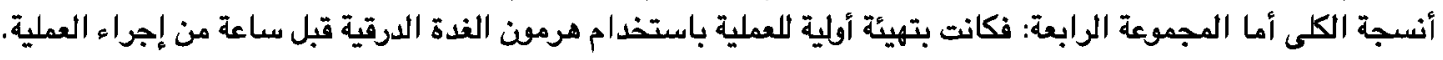

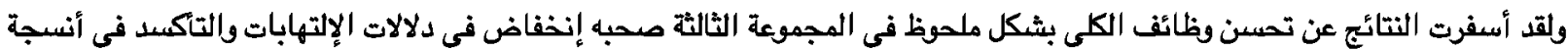

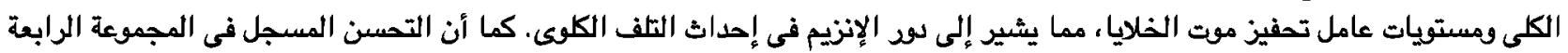

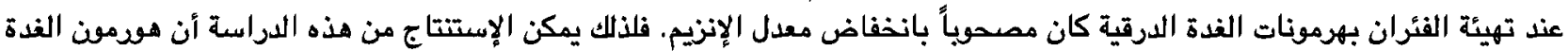

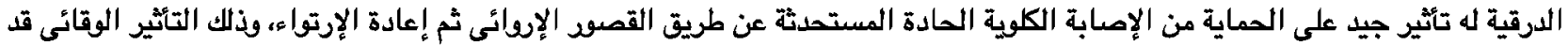

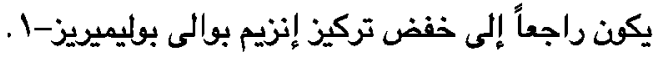

